\title{
Operating Penalties in Single-Fiber Operation 10-Gb/s, 1024-Way Split, 110-km Long-Reach Optical Access Networks
}

\author{
Darren P. Shea and John E. Mitchell
}

\begin{abstract}
We report for the first time optical signal-to-noise penalties which lead to performance degradations in single-fiber long-reach optical access networks when compared to identical dual-fiber systems. A simplified architecture, with reduced optical amplifier count compared to previous work, for single-fiber operation of a symmetrical 10-Gb/s, 1024-way split, 110-km long-reach optical access network is presented and demonstrated. In addition, a possible solution to remove the optical signal-to-noise penalty is suggested.
\end{abstract}

Index Terms-10 Gb/s, long-reach, next-generation access, optical access, single fiber.

\section{INTRODUCTION}

$\mathbf{L}$ ONG-REACH optical access networks represent the next step in the evolution of optical access networks which will deliver future high bandwidth services. Previous work in the area has reported the advantages and the technical feasibility of using such a network to provide a connection between the customer premises and the core network by extending the reach of the system to $110 \mathrm{~km}$ as opposed to the $20-\mathrm{km}$ reach of current passive optical networks (PONs) [1]. Further developments in optical technology, especially in the field of optical amplification, have enabled a simplification of the SuperPON architecture through the removal of the amplified splitter [2]. An increased split size of 1024 reduces the cost per user as the majority of the equipment is shared across all users. To ensure that each customer receives sufficient bandwidth, the data rate is set at $10 \mathrm{~Gb} / \mathrm{s}$, a four times increase over the current $2.488-\mathrm{Gb} / \mathrm{s}$ PONs. An additional capacity increase is also gained from statistical multiplexing, provided by increasing the number of customers to 1024. However, current PONs have an advantage in reducing the amount of installed fiber plant by using a single fiber to carry both upstream and downstream channels.

In this letter, we shall present a practical architecture which can be used to enable single-fiber operation for long-reach optical access networks. Single-fiber architectures have been suggested previously [3]. Our architecture presents an improvement by removing two erbium-doped fiber amplifiers (EDFA) from the backhaul section, which is realized by reducing the

Manuscript received June 29, 2006; revised September 27, 2006. This work was supported in part by the EPSRC and BT.

The authors are with the Department of Electronic and Electrical Engineering, University College London, London WC1E 7JE, U.K. (e-mail: dshea@ee.ucl.ac.uk; jmitchel@ee.ucl.ac.uk).

Color versions of Figs. 1-4 are available online at http://ieeexplore.ieee.org.

Digital Object Identifier 10.1109/LPT.2006.886848

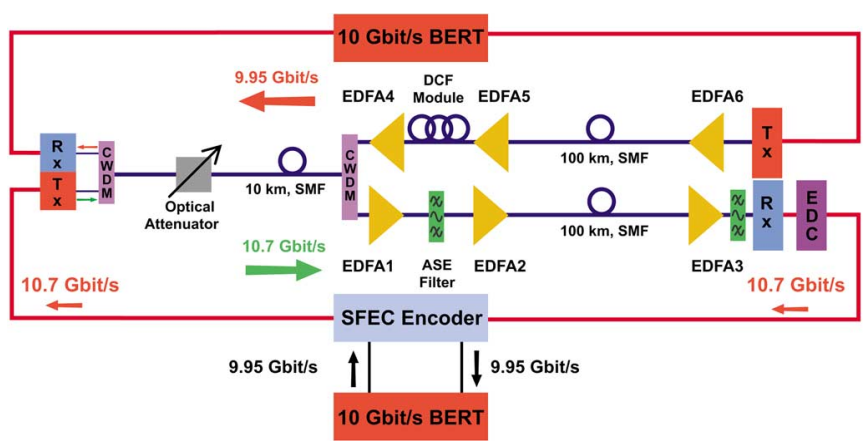

Fig. 1. Single-fiber distribution architecture. CWDM add-drop multiplexers are used to share the distribution section enabling the architecture to be an upgrade path for current PONs.

optical bandwidth to $2 \mathrm{~nm}$. The bandwidth reduction is enabled through the use of a multisource agreement compliant cooled transmitter which is envisioned to be extremely cost-effective through volume production as the device is suitable for a number of transmission formats including $10 \mathrm{G}$ Ethernet. In addition, for the first time, we identify and provide full explanation of an upstream penalty associated with single-fiber bidirectional transmission across a long-reach optical access network.

\section{EXPERIMENTAL SYSTEM}

A long-reach optical access network architecture for singlefiber operation from the customer premises to a core exchange is given in Fig. 1. This architecture uses separate EDFAs for the upstream and downstream transmission paths with coarse wavelength-division-multiplexing (CWDM) add-drop multiplexers to share the distribution section fiber. Existing PONs only provide connectivity between the customer premises and the local exchange where backhaul is provided through synchronous digital heirarchy networks. Hence, there is no requirement for the backhaul to be single-fiber, making the architecture given in Fig. 1 a practical solution to single-fiber operation for a longreach optical access network.

A laboratory demonstrator was built of the architecture given in Fig. 1. CWDM add-drop multiplexers (0.3-dB loss, 20-nm passband, centered around $1547 \mathrm{~nm}$ ) were placed at either end of the distribution section to allow the distribution section fiber to be used for both the upstream and downstream transmissions.

The distribution section was constructed from $10 \mathrm{~km}$ of standard single-mode fiber (SSMF) and a variable optical attenuator to account for split loss and additional loss associated with installed fiber. Conservative splitter losses $(3.5 \mathrm{~dB}$ per 
two-way split) are used with a fiber loss of $0.35 \mathrm{~dB} / \mathrm{km}$ plus optical flexibility point losses of 0.5 and $1 \mathrm{~dB}$ included at the customer premises and local exchange, respectively. Lumped attenuators are placed at regular intervals throughout each of the separate upstream and downstream 100-km backhaul SSMF spans to achieve the conservative fiber loss. Upstream, an EDFA preamplifier and an EDFA booster amplifier separated by an amplified spontaneous emission (ASE) filter (1532- to 1565-nm passband) provides gain $=46 \mathrm{~dB}$ and noise figure $=4.7 \mathrm{~dB}$. Downstream, a similar dual-stage EDFA arrangement is used with a high-power booster EDFA $\left(P_{\mathrm{SAT}}=21 \mathrm{dBm}\right)$. In the upstream transmission direction, $10.7-\mathrm{Gb} / \mathrm{s}$ continuous nonreturn-to-zero SuperFEC encoded data $(9.95 \mathrm{~Gb} / \mathrm{s}$ data $+7 \%$ overhead (see[4, Sec. I.7]) was applied to a $1555-\mathrm{nm}$ distributed feedback external modulated laser in a small form factor pluggable (XFP) casing. An optically preamplified receiver is used which consists of an EDFA with a 2-nm optical filter before a PIN photodiode. An electronic dispersion compensation (EDC) unit is placed after the upstream receiver to reduce the upstream dispersion penalty extending the reach of the upstream transmitter from 40 to $100 \mathrm{~km}$. The EDC unit is manually tuned but will be fully automatic in an installed system. EDC was chosen for its flexibility which allows end to end dispersion compensation for each individual optical network unit over the fully dynamic range of the PON compared with fixed length compensation provided by dispersion-compensating fiber. Downstream, a high-power transmitter is formed from a combination of a 1535-nm dense wavelength-division-multiplexing transmitter with a fully integrated $\mathrm{LiNbO}_{3}$ Mach-Zehnder modulator followed by an EDFA at the core exchange site. At the customer premises, the receiver section of the XFP transceiver is used. A dispersion-compensating fiber module proving compensation for $60 \mathrm{~km}$ of SSMF is positioned between the downstream EDFAs at the local exchange site to enable downstream transmission with the minimum dispersion penalty.

\section{Single-Fiber Operation PenAlty}

Experimental results for both the upstream and downstream paths are given in Fig. 2. Both directions exhibit performance degradation when compared with performance of the dual-fiber system (i.e., separate upstream and downstream fibers in both distribution and backhaul sections). Downstream, the system performance is thermal noise-limited as the ASE is heavily attenuated by the distribution section loss. Additional loss due to the introduction of CWDMs in the single-fiber system results in further signal attenuation and a degradation in optical signal-to-noise ratio (OSNR). Hence, the downstream experiences a performance degradation equivalent to the insertion loss of the CWDMs, i.e., $0.6 \mathrm{~dB}$.

It is clear from Fig. 2 that the upstream channel experiences greater performance degradation than the downstream. Further analysis of the upstream performance is given in Fig. 3 which quantifies the difference in the performance as $1.15 \mathrm{~dB}$, almost double the downstream performance degradation. Hence, further performance reducing effects must be present in the upstream path. Fig. 3 also illustrates influence of the downstream

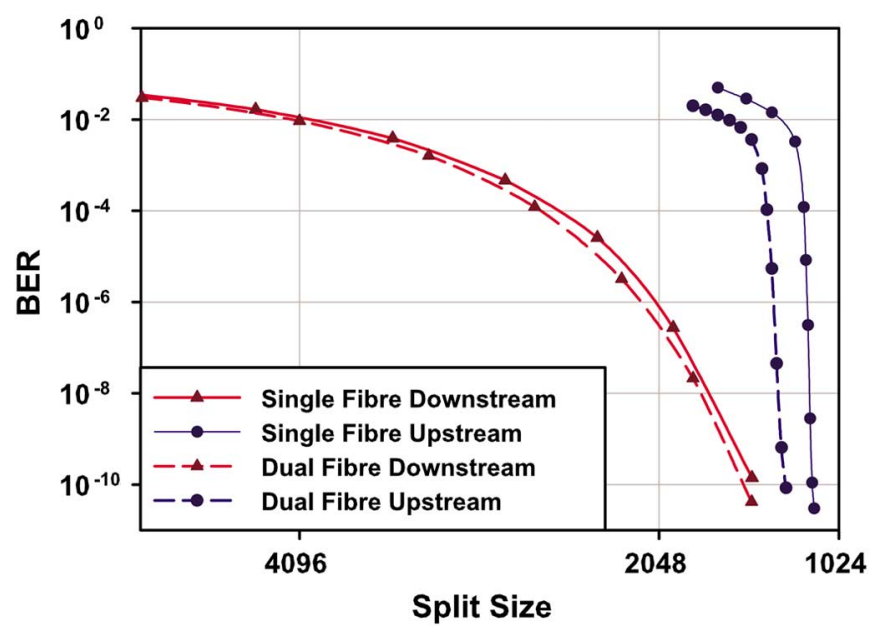

Fig. 2. Single-fiber operation BER performance measured against split size. A reduction in split size is seen in both transmission directions but is more severe in the upstream.

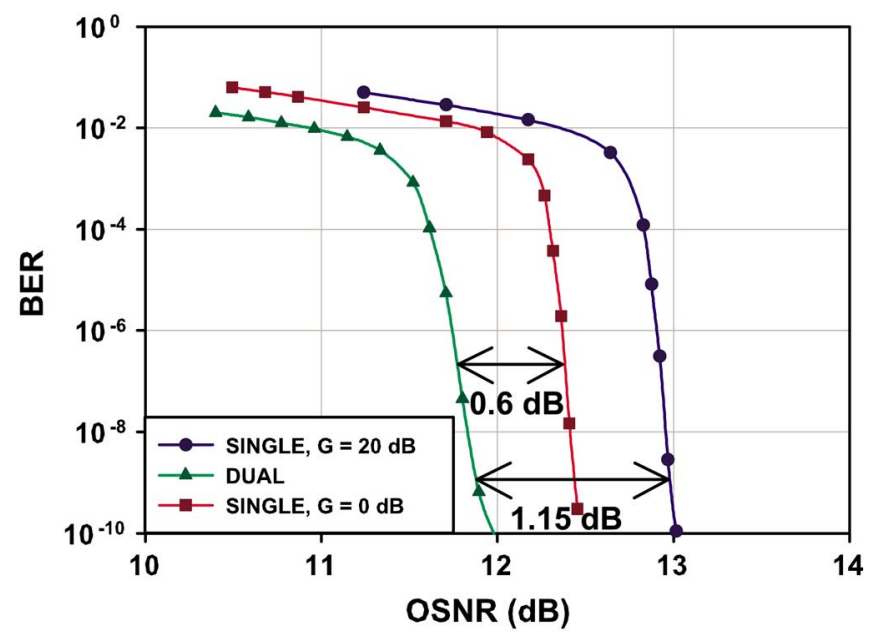

Fig. 3. OSNR penalty present in the upstream channel. An improvement in OSNR penalty is seen when the downstream gain is reduced.

transmission on the upstream transmission. The downstream path is unaffected by the presence of the upstream but it is clear that the downstream path reduces the performance of the upstream. As shown in Fig. 3, reducing the gain of the high-power EDFA in the downstream, from 20 to $0 \mathrm{~dB}$, reduces the upstream penalty to $0.6 \mathrm{~dB}$. This is the minimum penalty expected due to the insertion loss of the CWDMs, which demonstrates that the additional upstream penalty is due to the ASE in the downstream direction as a 0-dB gain would remove the downstream ASE. This suggests that the CWDM device positioned immediately after the downstream high-power EDFA introduces crosstalk between channels. Fig. 4 illustrates the detrimental effect of downstream ASE leaking through the CWDM coupler into the upstream path, using upstream and downstream spectra. In the lower left-hand corner of Fig. 4, the upstream signal spectrum shows that the upstream signal before the CWDM coupler is unaffected by the gain of the downstream EDFA4 gain. In the downstream direction, the CWDM coupler forms a notch filter which removes $18 \mathrm{~nm}$ of ASE generated by downstream EDFA4, between 1540 and $1560 \mathrm{~nm}$. 

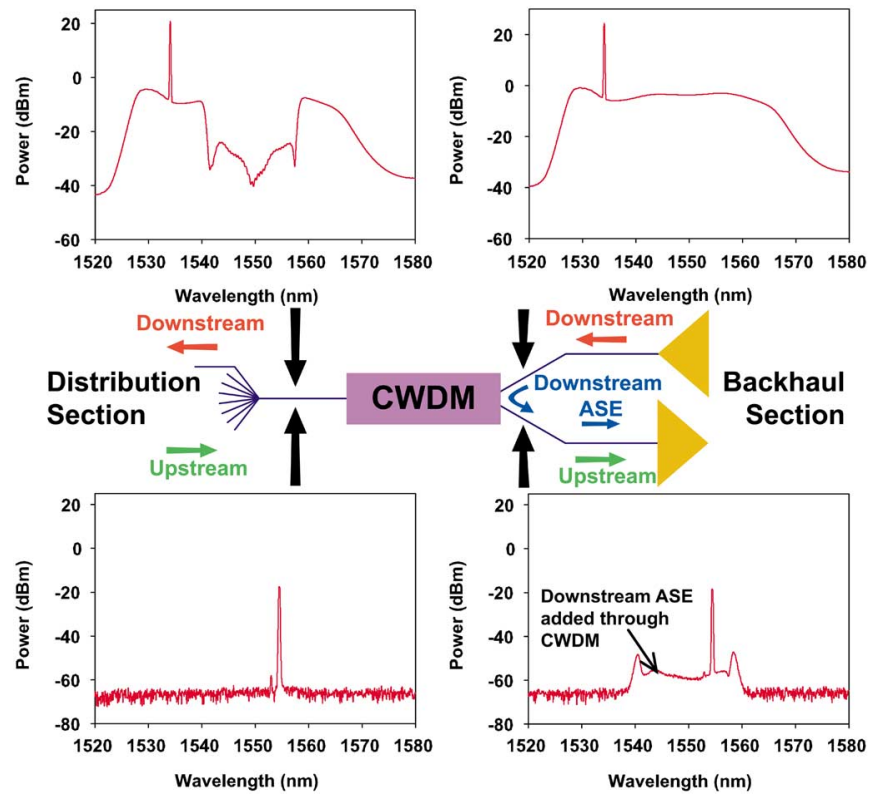

1520153015401550156015701580 Wavelength $(\mathrm{nm})$
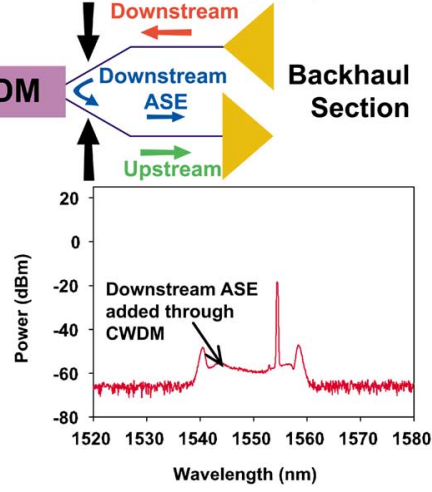

Fig. 4. Addition of downstream ASE into the upstream before EDFA1.

The isolation of the CWDM coupler between the upstream and downstream channels is $>50 \mathrm{~dB}$, which is good for such a device. However, due to the large difference between the upstream $(-36.3 \mathrm{dBm})$ and downstream signals (20 dBm), CWDM isolation is not great enough to suppress the downstream ASE to a level far below the upstream signal. Therefore, the upstream signal has an OSNR defined by the downstream ASE before the upstream amplification stages, seen in Fig. 4. The ASE injected into the upstream channel is low at $-58 \mathrm{dBm}(0.2-\mathrm{nm}$ bandwidth) when the gain of the high-power downstream EDFA4 is $20 \mathrm{~dB}$, the requirement for successful downstream operation. However, the additional noise is amplified by three EDFA stages, experiencing a net gain of $33 \mathrm{~dB}$, when the fiber and other losses are subtracted. Hence, the ASE contribution from the downstream ASE at the upstream receiver is significant.

Fig. 5 shows that the total noise at the upstream receiver is greater for the single-fiber system $(26.6 \mu \mathrm{W})$ than the dual-fiber system $(23.4 \mu \mathrm{W})$ due to the downstream ASE. Signal power levels for single- and dual-fiber systems were calculated analytically to determine the upstream OSNR for each system. A transmitter power of $4 \mathrm{dBm}$ was assumed which results in OSNRs of 11.85 and $13 \mathrm{~dB}$ at the receiver for single- and dual-fiber systems, respectively. Therefore, the 1.15-dB difference between the analytical OSNRs agrees with the experimental findings.

This additional penalty could be removed by incorporating an optical filter in the downstream path after EDFA4, before the CWDM coupler. A 10-nm filter centered on $1535 \mathrm{~nm}$ (downstream wavelength) would remove the ASE which overlaps with the upstream channel. Due to the thermal noise limit of the downstream, it is sensitive to any additional loss after EDFA4. If an ASE filter were to be incorporated, the gain and output power of EDFA4 would have to be increased to compensate for the loss of the filter to avoid detriment to the performance.

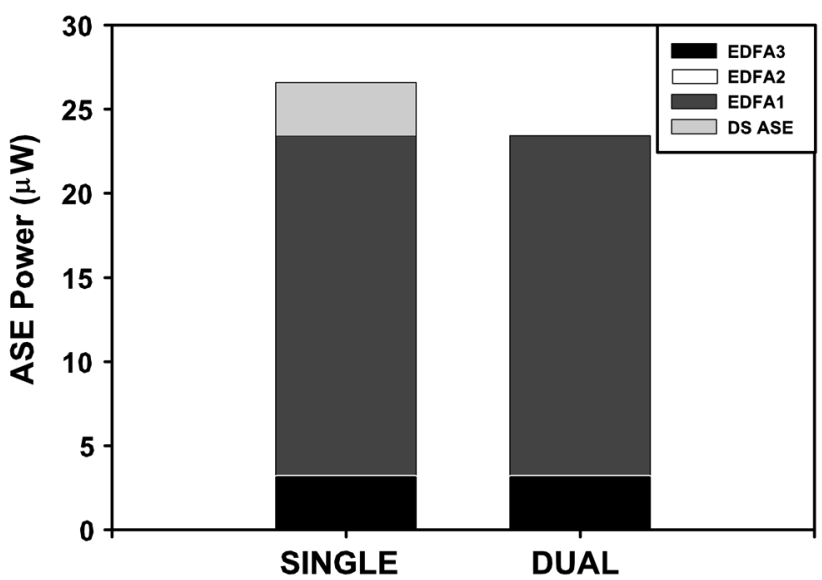

Fig. 5. ASE power at the upstream receiver for single- and dual-fiber systems EDFA1 provides the greatest ASE contribution and EDFA2 the smallest. In the single-fiber case, additional ASE is present increasing ASE at the receiver.

\section{CONCLUSION}

This letter furthered earlier work by constructing a singlefiber 1024-way split, 110-km 10-Gb/s long-reach optical access network with a reduced number of optical amplifiers compared with previous work, i.e., two EDFAs removed from the backhaul section. The target performance of bit-error rate $(\mathrm{BER})=$ $10^{-10}$ was achieved in both transmission directions. However, penalties were observed in both directions when compared with a dual-fiber network (i.e., no shared fiber). Downstream, the penalty was $0.6 \mathrm{~dB}$ which was attributed to the insertion loss of the CWDM couplers used to share the distribution section fiber. Upstream, a penalty of $1.15 \mathrm{~dB}$ was observed, $0.55 \mathrm{~dB}$ greater than the downstream penalty. The additional upstream penalty was due to ASE crosstalk across the CWDM coupler leaking from the downstream channel into the upstream channel. The ASE was amplified by the upstream transmission path causing an increase in ASE present at the receiver when compared with a dual-fiber system. A solution to this issues was suggested which involves incorporating a filter into the downstream EDFA4 to remove the ASE which overlaps with the upstream channel.

\section{ACKNOWLEDGMENT}

The authors would like to thank R. Davey and D. Nesset of BT for their support and facilities at Adastral Park.

\section{REFERENCES}

[1] I. Van de Voorde, C. M. Martin, I. Vandewege, and X. Z. Oiu, "The superPON demonstrator: An exploration of possible evolution paths for optical access networks," IEEE Commun. Mag., vol. 38, no. 2, pp. 74-82, Feb. 2000.

[2] D. P. Shea, A. D. Ellis, D. B. Payne, R. P. Davey, and J. E. Mitchell, "10 Gbit/s PON with $100 \mathrm{~km}$ reach and x1024 split," in Proc. ECOC 2003, Rimini, Italy, 2003, pp. 850-851, Paper We.P.147.

[3] D. Nesset, R. P. Davey, D. P. Shea, P. Kirkpatrick, S. Q. Shang, M. Lobel, and B. Christensen, "10 Gbit/s bidirectional transmission in 1024-way split, $110 \mathrm{~km}$ reach PON systems using commercial transceiver modules, SuperFEC and EDC," presented at the ECOC 2005, Glasgow, U.K., 2005

[4] Forward error correction for high bit rate DWDM submarine systems, ITU-T Recommendation G.975.1, 2004. 